\title{
An Interdisciplinary Guided Inquiry Laboratory for First Year Undergraduate Forensic Science Students
}

Sarah L Cresswell, * and Wendy A Loughlin

School of Natural Sciences, Griffith University, Brisbane, Queensland, 4111, Australia

ABSTRACT

An effective guided inquiry forensic case study (a pharmacy break-in) is described for first year students. Four robust introductory forensic chemistry and biology experiments are used to analyze potential drug samples and determine the identity of a possible suspect. Students perform presumptive tests for blood on a 'Point of Entry Stain' sample, chemical presumptive tests on the 'suspected drug' alongside known standards of codeine, morphine and amphetamine, and carry out TLC analysis of the drug samples. They examine the specificity of the Kastle-Meyer and tetramethylbenzidine tests, prepare PCR samples from the suspects' DNA samples and perform gel electrophoresis gel to analyze the results. Students are required to analyze and integrate the results and to apply their acquired knowledge within the context of an engaging forensic case study. This first year laboratory is part of a forensic case study vertically integrated into the curriculum.

\section{KEYWORDS}

First year Undergraduate/General; Interdisciplinary/Multidisciplinary, Laboratory Instruction; Hands-On Learning/Manipulatives, Inquiry-Based/Discovery Learning; Forensic Chemistry, Drugs/Pharmaceuticals, Qualitative Analysis, Electrophoresis, Thin Layer Chromatography.

The design of 'fit-for-purpose' forensic science degree programs of study has arisen in recognition of the need for an interdisciplinary approach to tertiary forensic science 
education. ${ }^{1-4}$ A solid grounding in science, in particular chemistry and biology, 5,6 in combination with the analytical and problem-solving skills for forensic applications of these scientific concepts is required for the training of forensic scientists. Articles describing how science can be related to forensic science, ${ }^{7-9}$ designed multi-technique forensic experiments, ${ }^{10}$ and describing an interdisciplinary approach, ${ }^{11-13}$ or an inquiry/problem based approach but in one field of science ${ }^{14}$ have been reported. Typically, these experiments (e.g. thin-layer chromatography (TLC) in combination with Gas Chromatography-Mass Spectrometry; 10 or Polymerase Chain Reaction (PCR) ${ }^{7,9}$ ) are focussed towards the technical knowledge and skills within one science discipline area, placing minor or no emphasis on the forensic context and interdisciplinary science knowledge required. The approach reported herein is a valuable addition to forensic science teaching as it introduces an integrated approach combining the introductory forensic science and forensic context with the interdisciplinary science from the start of a student's program of study in a first year subject.

We describe a guided inquiry ${ }^{15-17}$ approach as a mode for allowing students to undertake forensic case-based learning. ${ }^{18}$ Terminology of forensic case-based learning used herein will refer to guided inquiry learning carried out in a specific forensic context. A guided inquiry approach was used such that the problem, theory and procedures were presented to students, but the results analysis, results communication and conclusions are not provided. ${ }^{19}$ This suite of laboratory experiments was developed as forensic case-based learning in a similar way to expository labs ${ }^{20}$ in that the outcome is predetermined, however the outcome is unknown to the student, lending an element of problem solving unique to each group and each student. The intent of this approach to student learning was to: (a) integrate interdisciplinary scientific skills and techniques from chemistry, biology and forensic science and (b) use robust experiments and guided inquiry to facilitate student learning about forensic analysis and problem solving.

\section{PROCEDURE OVERVIEW}

The laboratory component of this semester-long subject comprised five 2-hour classes which were scaffolded by weekly 1-hour lectures and 1-hour tutorials. Students were introduced to the forensic case study via the Police Press Release (Figure 1) in their first lecture and then attended a laboratory class every second week for two hours. Students were placed in groups of three based initially on their degree program of study (single forensic science degree or double degree program of forensic science and criminology and criminal justice) and then on their chosen major; forensic chemistry or forensic 
molecular biology. This format has been successfully used for over three years for subject cohorts of 80-110 students. The comparison cohorts all studied the subject in the same semester of their program; where they were in their first year of study before choosing their major. The teaching team for the course remained constant over the three years.

The forensic case was as follows: At the scene of the break-in, a red stain was found on a broken window and this was submitted to students on filter paper which had been folded into a triangle shape; the tip of which is stained red/brown ('Point of Entry' Sample). Furthermore four suspects were identified by Police from CCTV footage and when the home they shared was visited by police and a clear liquid was seized from the scene for analysis as a potential illicit substance ('suspected drug' sample). Students were later provided with deoxyribonucleic acid (DNA) extracts from each of the four suspects to amplify and compare with the DNA from the point of entry stain.

The first laboratory class was run as a packaging and labelling tutorial. Students, in their groups, were given a selection of materials taken from fictitious crime scenes and they were asked to determine which are correctly, and which are incorrectly, packaged. This class allowed students to meet their group members, taught them the skills they will need to critique the packaging of the items they received during their case study, and introduced them to the importance of continuity of evidence. 


\section{POLICE PRESS RELEASE}

Four men, aged in their late twenties, have been detained on suspicion of committing the burglary of a pharmacist shop in Nathan last month. Police apprehended the men after they were identified from CCTV footage outside the shop.

The owner of the pharmacy stated that he arrived at his premises on Saturday $5^{\text {th }}$ July 2014 to discover a small window at the back of the premises had been broken and the lock forced. Upon entering the premises he saw several vials of medical supplies were missing and alerted police straight away.

When the police and scene of crime officers attended the scene they noticed there were drops of a red substance on the broken window and samples were collected for analysis.

A search of the suspects' home was undertaken on the $25^{\text {th }}$ July while the Police continued their questioning of the susnects.

Figure 1. Mock police press release provided to the students, which sets the scene for the forensic case study.

In the second laboratory class, each group was given two packages containing samples from the Pharmacy case; a 'Point of Entry Stain' sample along with the name and address of the pharmacy and the 'suspected drug' sample along with the address of the suspects. The samples in the packages, and addresses were different for each group of students on each given laboratory day.

The students performed the Kastle-Meyer $(\mathrm{KM})^{21}$ and tetramethylbenzidine (TMB) colour change tests for blood on the 'Point of Entry Stain' sample. The KM test was used to detect haemoglobin. The peroxidase activity of haemoglobin in blood catalyzes the oxidation of phenolphthalin (the colourless reduced form of phenolphthalein; reduced in situ in the KM reagent) by hydrogen peroxide into phenolphthalein, which has a strong pink colour under basic conditions. 3,3',5,5'-Tetramethylbenzidine acts as hydrogen 
donor for the reduction of hydrogen peroxide to water by the peroxidase enzymes, ${ }^{22}$ such as haemoglobin. Correspondingly TMB is oxidised to the tetramethylbenzidine diamine, and gave a positive reaction as a blue/green coloured solution.

Each blood presumptive test was first performed on a known blood sample before being repeated for a scraping of the 'Point of Entry Stain'. The students tested each sample with the KM or TMB reagents - no colour change was observed. Upon addition of hydrogen peroxide and in the presence of blood, the KM and TMB tests gave colour changes (see above) which indicated a positive reaction for the presence of blood.

The 'suspected drug' solution contained codeine, morphine or amphetamine (Fig 2) in methanol. The students performed chemical presumptive tests using both the Marquis ${ }^{23,24}$ and Mandelin ${ }^{25}$ colour tests. These tests were carried out on their 'suspected drug' alongside known standards of each of the three potential drugs to determine which was found at the suspects' address. In the Marquis test a purple colour indicates the presence of an opiate, such as codeine or morphine, and an orange/brown colour an amphetamine. ${ }^{24}$ In the Mandelin test, a reddish-brown colour was obtained for samples that are opiates and a green colour for amphetamines. Metavandate $\left(\mathrm{NH}_{4} \mathrm{VO}_{3}\right)$ is reduced (to $\mathrm{VO}_{2}{ }^{+}$in acidic solution) with concomitant oxidation of the opiate or amphetamine and produces a colour change with change in oxidation state of the vanadium. ${ }^{26}$
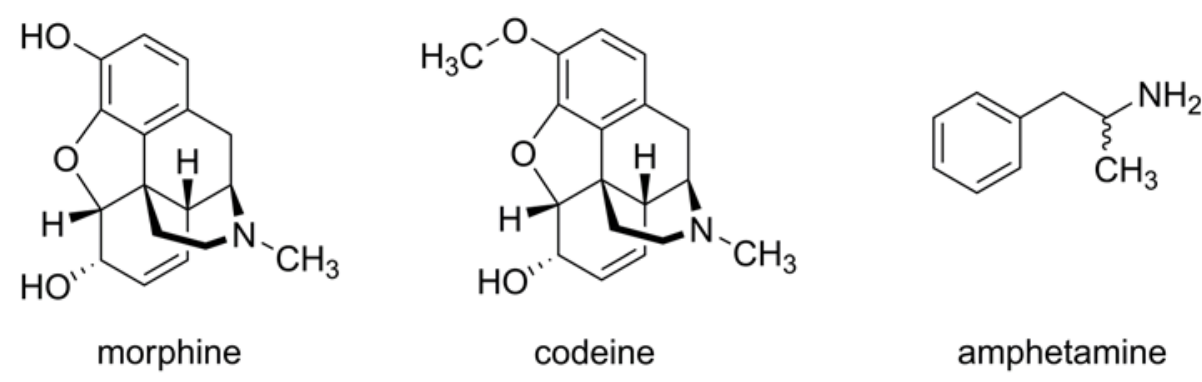

Figure 2. Chemical structures of morphine, codeine and amphetamine.

In the following laboratory session students performed TLC to support their presumptive test results, using the visualisation agents iodoplatinate (opiates) and ninhydrin (2,2-dihydroxyindane-1,3-dione) followed by iodoplatinate (amphetamines). ${ }^{27}$ Each group prepared two TLC plates with their 'suspected drug' to run alongside the single amphetamine, or two opiate, standards according to their previous results. Single 
and co-spotting techniques can be used. Students were able to confirm the results previously obtained from their colour change tests and can either comment on the reproducibility of the TLC system used (amphetamines) or comment on the suitability of each of the two opiate mobile phases used in this experiment.

In the fourth laboratory students first undertake dilution experiments using control blood samples to determine the limit of sensitivity of each of the two blood presumptive tests; the KM and TMB tests. In addition they were provided with four reference samples of DNA, one from each suspect, along with a sample of DNA which was extracted from their point of entry stain. The students prepared the five samples for the polymerase chain reaction (PCR). A PCR was carried out on the five samples in a thermal cycler during the interval ( 2 weeks) between the fourth and final laboratory session, which was supervised by a laboratory technician. By the final session, PCR products were available for use in the laboratory session.

In the final laboratory session students analysed their PCR products using electrophoresis; first making their own gel. Their samples were run on a gel electrophoresis apparatus alongside an allelic ladder to separate the PCR products. The gels were visualised under UV light and photographed for the students. The results for the PCR products were then compared to the simulated ladder of possible alleles and a genotype was assigned. Analysis was carried out to determine whether any of the four suspects' genotype matched the crime scene sample.

A detailed description of the experiments is in the supporting information along with laboratory objectives, student handouts and instructions and hazards and safety precautions.

\section{MATERIALS}

The 'Point of Entry Stain' sample is bovine haemoglobin on filter paper. The DNA samples are from the PCR kit, which uses non-human DNA sources. The 'Suspected drug' sample' is a $1 \mathrm{mg}$ in $1 \mathrm{~mL}$ solution of a controlled drug substance in methanol of codeine, morphine or amphetamine. The presumptive test reagents are; KM reagent (phenolphthalein, potassium hydroxide, distilled water, zinc), TMB reagent (tetramethylbenzidine in glacial acetic acid), hydrogen peroxide $(3 \% \mathrm{w} / \mathrm{v})$, Mandelin reagent (ammonium vanadate $\left(\mathrm{NH}_{4} \mathrm{VO}_{3}, 1.0 \mathrm{~g}\right)$ in concentrated sulfuric acid $(100 \mathrm{~mL})$, Marquis reagent (formaldehyde (40\%), concentrated sulfuric acid). A Crime Scene Investigator PCR Basics Kit can be purchased and has required accessories including a 
microcentrifuge, thermal cycler, power supply, and horizontal gel electrophoresis system with mini caster.

Hazards

Ethyl acetate, methanol, and ethanol are flammable. Chloroform is toxic and a carcinogen. Ammonia is corrosive and an irritant. Hydrochloric acid and sulfuric acid are caustic and should not be inhaled. KM and Mandelin test reagents, iodoplatinate and ninhydrin sprays are toxic and harmful if swallowed. Ethidium Bromide is a potent mutagen, toxic and harmful if swallowed, touched or inhaled. Gloves, goggles/safety glasses, closed-in protective shoes, and a protective laboratory coat must be worn. All operations should be performed in well ventilated areas, such as fume hoods.

Licencing requirements

Jurisdictional differences will exist but in Queensland the procurement and use of codeine, morphine and amphetamine is controlled by the Drugs Misuse Act 1986 and the Drugs Misuse Regulation 1987; in the USA it is controlled by the United States Drug Enforcement Agency and Controlled Substances Act. A licence must be obtained from the relevant authority before these materials can be purchased. Once obtained they must be stored in a locked drugs safe and records must be kept of how much is purchased and subsequently used such that an audit trail can be created for each illicit material. Disposal of drug contaminated samples was carried out via secure waste disposal services of solvents or packaged solids, which are incinerated.

\section{STUDENT RESULTS}

Initially the students determined that the point of entry stain contained blood and that the colourless liquid 'suspected drug' samples from the home of the suspects contained one of the three drugs known to have been stolen from the pharmacy. The blood presumptive tests work more slowly with synthetic haemoglobin than they do with whole blood so students needed to be told not to expect the instantaneous colour changes they see on television. Horseradish can be included as a false positive (subject to availability), as it contains peroxidase. The tests worked well and students obtained clear and repeatable results (Table 1). 
Table 1. Student Results ${ }^{a}$ from the Blood Presumptive Tests

\begin{tabular}{|c|c|c|c|c|}
\hline Sample & $\begin{array}{l}\text { Colour Change } \\
\text { After TMB }\end{array}$ & $\begin{array}{l}\text { Colour Change } \\
\text { After Addition of } \\
\text { Hydrogen Peroxide }\end{array}$ & $\begin{array}{l}\text { Colour Change } \\
\text { After KM Addition }\end{array}$ & $\begin{array}{l}\text { Colour Change } \\
\text { After Addition of } \\
\text { Hydrogen Peroxide }\end{array}$ \\
\hline $\begin{array}{l}\text { Control - } \\
\text { blood }\end{array}$ & No change & Green-yellow & No change & Pink \\
\hline $\begin{array}{l}\text { Point of } \\
\text { entry stain }\end{array}$ & No change & Green-yellow & No change & Pink \\
\hline Grass & No change & No change & Light green-yellow & Pink around edge \\
\hline Rust & No change & No change & No change & Pink \\
\hline Banana & No change & No change & No change & Pink around edge \\
\hline Horseradish & No change & Green-yellow & No change & Pink around edge \\
\hline
\end{tabular}

${ }^{a}$ Typical observations recorded by students.

Typical observations by students, from a set of chemical presumptive tests is shown in Table 2, where the student correctly identified the drug in this experiment as an opiate, consistent with it being codeine.

Table 2. Student Results ${ }^{a}$ from the Chemical Presumptive Tests

\begin{tabular}{|c|c|c|c|c|}
\hline \multirow[b]{2}{*}{ Sample } & \multicolumn{2}{|c|}{ Immediate Colour Change } & \multicolumn{2}{|c|}{ Colour Change after 5 minutes } \\
\hline & Marquis & Mandelin & Marquis & Mandelin \\
\hline Morphine & Pink & Brown & Pink/purple & Dark brown/purple \\
\hline Amphetamine & Orange & $\begin{array}{l}\text { Yellow - no } \\
\text { change }\end{array}$ & Burnt orange & Green tinge \\
\hline Codeine & Purple & Green & Purple/blue & Dark green/brown \\
\hline $\begin{array}{l}\text { Suspected } \\
\text { Drug }\end{array}$ & Purple & Green & Purple/blue & Dark green/brown \\
\hline Control & - & Yellow & - & Yellow \\
\hline
\end{tabular}

${ }^{a}$ Typical observations recorded by students.

Some groups of students were less confident in their colour change results for the 'suspected drug' presumptive test analysis than others primarily because they added the test reagent to the dilute drug sample without allowing sufficient time for the solvent to evaporate. Student groups who followed the directions of the lab staff regarding evaporation of solvent obtained very clear results and did not need to repeat 
this activity. Student attempts at labelling the spotting tiles with a marker pen resulted in discolouration of the solutions in the wells as marker pen ink is soluble in methanol. Students were advised to draw and label a diagram of their spotting tile in their lab book rather than attempt to label the tile itself with ink.

The tentative confirmation of drug classes was achieved using TLC (Tables 3 and 4). The students confirmed that their 'suspected drug' was an opiate and, from the TLC results correctly identified it to contain codeine.

Table 3. Student Results ${ }^{a}$ from Thin Layer Chromatography Analysis of 'Suspected Drug' Sample

\begin{tabular}{lcccc} 
& \multicolumn{2}{c}{ Solvent $1^{b}$} & \multicolumn{2}{c}{ Solvent $2^{c}$} \\
\cline { 2 - 5 } Sample & 2 & $R_{f}$ & $\begin{array}{c}\text { Distance moved by } \\
\text { component }(\mathrm{mm})\end{array}$ & $R_{f}$ \\
Morphine & 15 & 0.03 & 12 & 0.18 \\
Component $(\mathrm{mm})$ & 0.22 & 25 & 0.38 \\
Suspected drug & 15 & 0.22 & 25 & 0.38 \\
Solvent 1 Front & 68 & 1.00 & 65 & 1.00
\end{tabular}

${ }^{a}$ Typical observations recorded by students. ${ }^{b}$ In this example solvent 1 is chloroform:methanol (9:1, v/v). ${ }^{c}$ In this example solvent 2 is ethyl acetate:methanol:ammonia (85:10:5, v/v).

Table 4. Student Results ${ }^{a}$ of Visualization of Thin Layer Chromatography Analysis of 'Suspected Drug' Sample

\section{TLC test observation stage}

Under normal lighting conditions

Under 254 nm UV light (light box)

Sprayed with acidified iodoplatinate solution

Solvent $1^{b}$

No visible spots

Two very pale, light pink/purple spots visible approximately half way up the TLC plate

Background of TLC was an almost uniform light orange colour. Two purple spots and one blue spot were visible on the TLC plate. The blue spot had not travelled far from the baseline.

\section{Solvent $2^{c}$}

No visible spots

Two very pale, light pink/purple spots visible approximately half way up the TLC plate

Background of TLC was an almost uniform pink colour. Two purple spots and one blue spot were visible on the TLC plate. The blue spot had travelled a shorter distance than the two purple spots.

\footnotetext{
${ }^{a}$ Typical observations recorded by students. ${ }^{b}$ In this example solvent 1 is chloroform:methanol $(9: 1, \mathrm{v} / \mathrm{v}) .{ }^{c}$ In this example solvent 2 is ethyl acetate:methanol:ammonia (85:10:5, v/v).
}

Next, amplification and analysis of the point of entry stain, in comparison with DNA extracts from each of the four suspects, was undertaken (Figure 3). The use of bovine haemoglobin in the PCR kit eliminates the possibility of human DNA contamination and Biohazard classification in the undergraduate laboratory setting.

The PCR test kit used allowed the students to generate products for analysis by electrophoresis. All vials needed to be sealed before the PCR process was undertaken otherwise sample loss was an issue. This was the first opportunity for students to 
undertake gel electrophoresis and some students found the task of adding their samples to the wells with a pipette very difficult. Some of the students' loaded more than one sample in one well and/or had some merging of lanes due to incorrect technique in preparing and loading the gel for analysis.

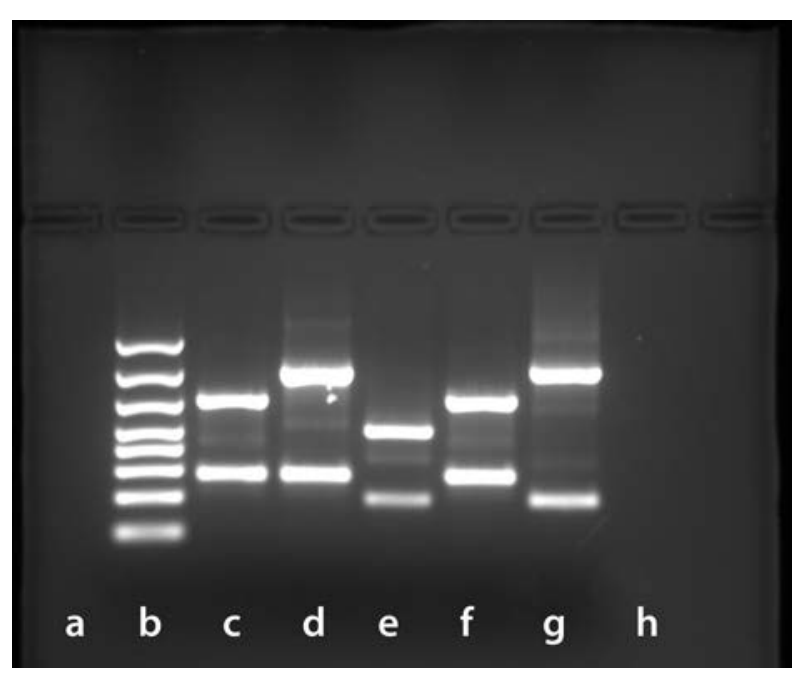

Figure 3. Electrophoresis gel photographed under UV light depicting the results from a representative group of students for the experiment to determine the donor of the red bloodstain found at the point of entry at the pharmacy break-in. Key: From left to right the lanes in the gel represent: (a) blank; (b) allele ladder; (c) point of entry stain; (d) Arthur Bravo; (e) Christopher Delta; (f) Edward Foxtrot; (g) Gareth Hotel; (h) blank

Each laboratory session was assessed by means of a group laboratory report in which each team member was responsible for a specific section of the report and the responsibility for each section was rotated amongst the team members for subsequent reports. Each report was marked and returned within 1 week. During the laboratories biology experiments (blood presumptive tests, PCR and electrophoresis) and chemistry experiments (chemical presumptive tests, and TLC analysis) were linked in an interdisciplinary approach within the forensic case study, which required student to correlate, link and contextualise the results from each experiment and present the conclusions (statement of witness) for the forensic case. The students' laboratory reports clearly demonstrated that the students had met the objectives of each laboratory session. Students were able to discuss (in a written form) the chemical and biology results and go further to explain what the results meant within the context of 
the forensic case, and draw valid forensic conclusions. Once all the interdisciplinary scientific information was available, each student prepared a final report; a statement of witness about their involvement in the case suitable for presentation in a Queensland Court of Law.

\section{INFORMAL FEEDBACK}

Student feedback on this course was generally very positive. In the first iteration we had groups of up to four students and this, in some cases, lead to issues with students who did not fully participate in either the laboratory sessions or in the preparation of the laboratory report. In subsequent years group sizes were reduced to a maximum of three people and although some students still do not like group work, the amount of negative feedback relating to group size and participation of group members has dramatically reduced. Anecdotally, students have commented that they prefer smaller member group size (such as three) as it provides less opportunity for group members to opt out of work contributing to the group effort. The end of semester evaluations in all of the guided inquiry years were in the range of $70-85 \%$ overall satisfied with the course (4 years, 300 students). Students made positive comments such as, the labs were "a lot of fun and helped piece together what I had heard in lectures and tutorials" and the labs "helped us to understand how a forensic scientist works". The laboratory and witness statement were incorporated as assessment items worth $40 \%$, with all other types of assessment items (mid-semester quiz, end of semester exam) remaining unchanged apart from the assessment weighting. In this context, it was interesting to note that the overall performance of students was better (subject average mark $67 \pm 7 \%$ ) when compared to previous cohorts who did not undertake the guided inquiry laboratory sessions (subject average mark 62 $\pm 13 \%$ ).

\section{DISCUSSION AND CONCLUSIONS}

Guided inquiry ${ }^{15-17}$ and partial guided inquiry has been shown to be effective for improved student achievement. ${ }^{28}$ The interdisciplinary guided inquiry approach used here required students to solve the forensic case scenario by analysing multiple evidence samples in the laboratory sessions, by understanding the sciences of molecular biology and chemistry, by developing scientific experimental and investigation skills as well as higher-order evaluation and conclusion skills. A multievidence approach using underpinning science which was readily accessible for a 
foundation course in $1^{\text {st }}$ year drove the choice of the pharmacy break-in scenario presented to the students.

Prior to the laboratory sessions, students had no hands-on exposure of the different chemical and biology techniques relevant to forensic analysis. The laboratory experiments were robust, relatively easy to perform, had visual results and produced obvious results with clear conclusions. The results obtained by each group of students were unique to that group and this was achieved by having 4 potential suspects, 6 different pharmacies and suspects' addresses and 3 possible illicit substances stolen. The visual experimental results allowed students to make and test hypotheses regarding the drug present and the identification of the possible suspect. By completing these laboratories the students acquired a range of relevant techniques (presumptive tests, TLC, electrophoresis, PCR) and started to integrate forensic chemistry and biology concepts and techniques within the context of a forensic case scenario. Students were able to draw on their conclusions from each laboratory session in their laboratory reports and to determine an effective forensic appraisal of the evidence for the pharmacy break-in forensic case.

The students were required to work in teams, but some students gave feedback that they did not like group work. However, grouping students with biology or chemistry interests to write group laboratory reports was intended to be reflective of the professional team environment of forensic scientists, and enabled students to make an informed choice about which major, forensic chemistry or forensic molecular biology, to pursue during their degree program.

The final report (witness statement) completed by each student individually, gave details and a description of the crime scene samples provided, results of the chemical and biological tests, and presented the key conclusions of the nature of the drug and the identification of the blood stain. Students were provided tutorial sessions to discuss the requirements of such a document. The practice statement produced was extensively annotated during marking so that students could improve their statement before revisiting the case in a subsequent year in an Expert Witness course (This laboratory can stand on its own or as part of a vertically integrated forensic science curriculum). Requiring the students to write up the laboratories as a Statement of Witness, in addition to conventional scientific reports, challenged the students to provide the forensic evidence in a non-biased way, and not overthink the evidence, extrapolate the results or jump to conclusions. 
In conclusion, students' feedback to the partial guided inquiry approach within laboratories was overwhelmingly positive, with the exception of comments relating to group work. Feedback confirmed that the laboratories are pitched at an appropriate level for first-year students. The partial guided inquiry approach presented herein used an interdisciplinary approach with structured assessment (reports and witness statement) to develop in students, theoretical and technical skills for an integrated suite of chemical and biological experiments, skills to interpret results within a forensic context and reach valid forensic conclusions. In doing so, students were introduced to forensic techniques and analysis, assisting them in informed choices for their future careers in forensic science.

\section{ASSOCIATED CONTENT}

Supporting Information

Laboratory objectives. Experimental procedures for blood presumptive tests, chemical presumptive tests, thin-layer chromatography, PCR and electrophoresis. A student handout for the PCR procedure instructions; A student handout, including safety instructions and potential hazards for the preparation of the electrophoresis gel. Instructions for Laboratory Reports. Hazards and Safety Precautions.

\section{AUTHOR INFORMATION}

Corresponding Author

*E-mail: w.loughlin@griffith.edu.au

Notes

The authors declare no competing financial interest.

\section{ACKNOWLEDGMENTS}

The authors would like to thank Alex Forrest and Kirsty Wright for collegial discussion. This work was supported by a Griffith University Grant for Learning and Teaching. Human Ethics approval number BPS/12/10/HREC

\section{REFERENCES}

1. Lewis, S.; Brightman, R.; Roux, C. Forensic Science Tertiary Education in Australia. Chem. Australia. 2005, 72(3), 4-8. ISSN: 0314-4240.

2. Mennell, J. The Future of Forensic and Crime Scene Science. Part II. A UK Perspective on Forensic Science Education. Forensic Sci. Int. 2006, 157S, S13-S20. 
3. Adams, D. E.; McCoy, M.; Jourdan, T.; Lord W. Forensic Science Education Programs: A New Paradigm. Sci. Justice. 2010, 50, 26.

4. Quarino, L.; Brettell, T. A. Current Issues in Forensic Science Higher Education, Anal. Bioanal. Chem. 2008, 394, 1987-1993.

5. Almirall, J. R.; Furton, K. G. Trends in Forensic Education: Expansion and Increased Accountability. Anal. Bioanal. Chem. 2003, 376, 1156-1159.

6. Robertson, J.; Roux, C. The Development and Enhancement of Forensic Expertise: Higher Education and In-service Training in Handbook of Forensic Science, Fraser, J.; Williams, R. Ed.; Willan Publishing: USA and Canada, 2009; pp 566-595. ISBN-978-1-84392-312-1

7. Millard, J. T.; Chuang, E.; Lucas, J. S.; Nagy, E. E.; Davis, G.T. Case-Study Investigation of Equine Maternity via PCR-RFLP: A Biochemistry Laboratory Experiment. J. Chem. Ed. 2013, 90, 1518-1521.

8. Schurter, E. J.; Zook-Gerdau, L. A.; Szalay, P. Analysis of a Suspected Drug. J. Chem. Ed. 2011, 88, 1416-1418.

9. Elkins, K. M.; Kadunc, R. E. An Undergraduate Laboratory Experiment for Upper-Level Forensic Science, Biochemistry or Molecular Biology Courses: Human DNA Amplification Using STR Single Locus Primers by Real-Time PCR with SYBR Green Detection. J. Chem. Ed. 2012, 89, 784-790.

10. Szalay, P. S.; Zook-Gerdau, L. A.; Schurter, E. J. A Multi-Technique Forensic Experiment for a Nonscience-Major Chemistry Course. J. Chem. Ed. 2011, 88, 1419-1421.

11. Marle, P. D.; Decker, L.; Taylor, V.; Fitzpatrick, K.; Khaliqi, D.; Owens, J. E.; Henry, R. M. CSI-Chocolate Science Investigation and the Case of the Recipe Rip-Off: Using an extended Problem-Based Scenario to enhance High School Students' Science engagement. J. Chem. Ed. 2014, 91, 345-350.

12. Friesen, J. B. Forensic Chemistry: The revelation of Latent Fingerprints. J. Chem. Ed. 2015, 92, 497-504.

13. Heimbuck, C. A.; Bower, N. W. Teaching Experimental Design Using a GC-MS of Cocaine on Money; A Cross-disciplinary Laboratory. J. Chem. Ed. 2002, 79, 1254-1256.

14. Maurer, M. K.; Bukowski, M. R.; Menachery, M. D.; Zatorisky, A. R. Inquiry-based Arson Investigation for General Chemistry using GC-MS. J. Chem. Ed. 2010, 87, 311-313.

15. Baseya, J. M; Francis, C.D. Design of Inquiry-Oriented Science Labs: Impacts on Students' Attitudes. Res. Sci. Tech. Ed. 2001, 29(3), 241-255.

16. Chatterjee, S.; Williamson, V. M.; McCann, K.; Peck, M. L. Surveying Students' Attitudes and Perceptions Towards Guided Inquiry and Open Inquiry Laboratories. Chem. Ed. Res. 2009, 86(12), 1427-1432.

17. Allen, J. B.; Barker, L. N.; Ramsden, J. H. Guided Inquiry Laboratory. J. Chem. Ed. 1986, $63,533-534$. 
18. Summerfield, S.; Overton, T. Problem-Solving Case Studies. Anal. Chem. 2003, 75(7), 181A-182A.

19. Buck, L. B; Bretz, S. L.; Towns, M. H. Characterizing the Level of Inquiry in the Undergraduate Laboratory. J College Sci. Teach. 2008, 38, 52-58.

20. Domin, D. S. A Review of Laboratory Instruction Styles. J. Chem. Ed. 1999, 76, 543-547.

21. Glaister, J.; The Kastle-Meyer Test for the Detection of Blood, Brit. Med. J. 1926, 650652.

22. Garner, D. D.; Cano, K. M.; Peimer, R. S.; Yeshion, T. E. An Evaluation of Tetramethyl benzidine as a Presumptive Test for Blood. J. Forensic Sci. 1976, 21(4), 816-821.

23. James, R. E.; Saferstein, R.; Meloan, C. E. Lab Manual, Criminalistics: An Introduction to Forensic Science, 7th ed.; Pearson Prentice: Upper Saddle River, NJ, 2001.

24. Anderson, C. Presumptive and Confirmatory Drug Tests. J. Chem. Ed. 2005, 82, 18091810.

25. Fike, W. W.; Sunshine I. Identification of Antihistamines in Extracts of Biological Materials Using Thin Layer Chromatography. Anal. Chem. 1965, 37, 127-129.

26. Spangenberg, B.; Poole, C.F.; Weins, Ch. Quantitative Thin-Layer Chromatography: A Practical Survey. Springer: Heidelberg, 2011. pp172.

27. Dutt, M. C.; Poh, T. T. Use of Ninhydrin as a Spray Reagent for the Detection of Some Basic Drugs on Thin-Layer Chromatograms. J. Chromat. 1980, 195, 133-138.

28. Conway, C. J. Effects of Guided Inquiry versus Lecture Instruction on Final Distribution in a One-Semester Organic and Biochemistry Course. J. Chem. Ed. 2014, 91, 480-483.

\section{For Table of Contents Only}

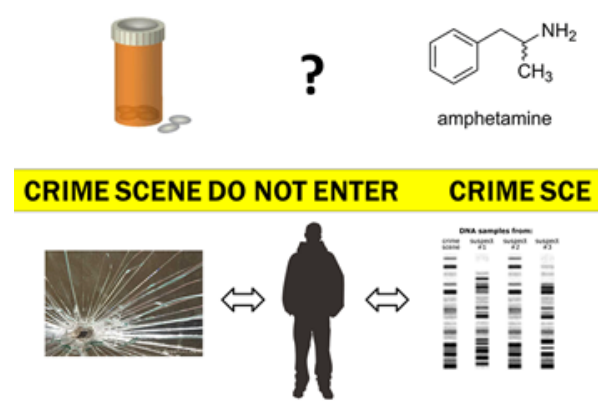

